\title{
DNA Methylation Signature of Post-injury Neointimal Cells During Vascular Remodeling in the Rat Balloon Injury Model
}

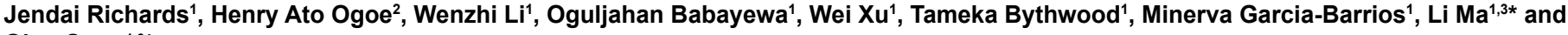 \\ Qing Song ${ }^{1,3 *}$ \\ ${ }^{1}$ Cardiovascular Research Institute and Department of Medicine, Morehouse School of Medicine, Atlanta, Georgia, USA \\ ${ }^{2}$ Department of Biomedical Informatics, University of Pittsburgh, Pittsburgh, Pennsylvania, USA
}

${ }^{3} 4$ DGenome Inc, Atlanta, Georgia, USA

\begin{abstract}
Vascular smooth muscle cell (VSMC) accumulation in the neointimal is a common feature in vascular diseases such as atherosclerosis, transplant arteriosclerosis and restenosis. In this study, we isolated the neointimal cells and uninjured residential vascular smooth muscle cells by laser micro dissection and carried out single-cell whole-genome methylation sequencing. We also sequenced the bisulfite converted genome of circulating bone-marrow-derived cells such as peripheral blood mononuclear cells (PBMC) and bone marrow mononuclear cells (BMMC). We found totally 2,360 differential methylation sites (DMS) annotated to 1,127 gene regions. The majority of differentially methylated regions (DMRs) were located in intergenic regions, outside those CpG islands and island shores. Interestingly, exons have less DMRs than promotors and introns, and CpG islands contain more DMRs than islands shores. Pearson correlation analysis showed a clear clustering of neointimal cells with PBMC/BMMC. Gene set enrichment analysis of differentially methylated $\mathrm{CpG}$ sites revealed that many genes were important for regulation of VSMC differentiation and stem cell maintenance. In conclusion, our results showed that neointimal cells are more similar to the progenitor cells in methylation profile than the residential VSMCs at the $30^{\text {th }}$ day after the vascular injury.
\end{abstract}

Keywords: Biomarkers; DNA methylation; Atherosclerosis

\section{Introduction}

Vascular smooth muscle cell (VSMC) accumulation in the neointima is a common feature in vascular diseases such as atherosclerosis, transplant arteriosclerosis, and restenosis. In all of the phenomena, one of the characteristic changes is the accumulation of VSMCs within the neointima. The contemporary paradigm explaining the pathophysiology of neointima formation is that residential VSMCs dedifferentiate, proliferate and migrate from the media to form the neointimal cells $[1,2]$. The apparent increase in proliferation among the tunica media cells after injury [3-6] and the ex vivo development of neointima in arterial culture systems [7-9] supported this theory. It was further reinforced by the fact that neointimal cells expressed those VSMC markers, such as smooth muscle alpha actin (SMA), and vimentin [10-12]. The classical paradigm has been challenged by Sata et al who proposed that neointimal cells in atherosclerotic arteries are derived from bone marrow (BM) derived circulating progenitor cells [13-15]. It was reported that $>80 \%$ of SMC-derived cells within advanced atherosclerotic lesions could not be detected by traditional immunostaining-based methods [16]; furthermore, these unidentified SMC-derived cells exhibit phenotypes of other cell lineages, including macrophages and mesenchymal stem cells (MSCs) [16]. Because the key features on the expression of SMC-selective gene products, such as SM MHC and SM $a$-actin (SMaA) have been lost, it is controversial about the definitive identification or origin of VSMCs during vascular remodeling [17-20]. DNA methylation is one of the major epigenetic modifications in mammalian genomes; it directly dictates the decision-making during differentiation, lineage stability and cellular phenotypes in progeny cells [21-24]. Recent studies have shown that iPSC can remember their origins with their memory implemented into the DNA methylations at certain loci after reverse differentiation [25-29]. This is important because from where neointimal cells in atherosclerosis migrated, the vascular smooth muscle cells or VSMC-like cells in the neointima or atherosclerosis may all have the same protein markers; some markers are even present on the surface of circulating progenitor cells, local progenitor cells, dedifferentiated VSMCs, and regular VSMCs. Because the differentiated cells may still remember their origins implemented in their memory via DNA methylation patterns, we sought to compare the DNA methylation profiles between neointimal cells, residential VSMCs and bone marrow derived circulating progenitor cells.

\section{Methods}

\section{Balloon injury model and tissue collection}

Wild-type (WT) inbred Sprague Dawley rats were purchased from Charles River Laboratories. Animal procedures were approved by the Institutional Committee for Use and Care of Laboratory Animals at Morehouse School of Medicine and conform to the Guide for the Care and Use of Laboratory Animals published by US National Institutes of Health. Male Sprague-Dawley rats of 6-10 weeks old were anesthetized with isoflurane. The left carotid artery was exposed via a vertical midline incision and an arteriotomy was performed. The left common carotid artery was injured with an embolectomy balloon catheter $(2 \mathrm{~F}$ Fogarty, Edwards Life Sciences) by inflating to 1.5 atmospheres and retracting to the arteriotomy site three times to assure a good vascular injury [30]. The wound and skin were closed with absorbable and non-absorbable sutures, respectively. Thirty days after injury, rats were euthanized with overexposure to $\mathrm{CO}_{2}$, blood was collected from the heart via intra-cardiac puncture; and the injured and normal arteries were exposed, arteries were collected and fixed in $4 \%$ formalin. Arteries were embedded in the paraffin block, cut into $10-\mu \mathrm{m}$ sections, and then

*Corresponding authors: Qing Song, Cardiovascular Research Institute Morehouse School of Medicine, 720 Westview Drive SW, Atlanta, GA 30310, USA Tel: 404-752-1845, E-mail: qsong@msm.edu

Li Ma, Cardiovascular Research Institute, Morehouse School of Medicine, 720 Westview Drive SW, Atlanta, GA 30310, USA, Tel no: 404-752-1845, Email: Ima@msm.edu

Received May 04, 2016; Accepted May 11, 2016; Published May 18, 2016

Citation: Richards J, Ogoe HA, Li W, Babayewa O, Xu W, et al. (2016) DNA Methylation Signature of Post-injury Neointimal Cells During Vascular Remodeling in the Rat Balloon Injury Model. Mol Biol 5: 163. doi:10.4172/2168-9547.1000163

Copyright: ( $) 2016$ Richards J, et al. This is an open-access article distributed under the terms of the Creative Commons Attribution License, which permits unrestricted use, distribution, and reproduction in any medium, provided the original author and source are credited. 
Citation: Richards J, Ogoe HA, Li W, Babayewa O, Xu W, et al. (2016) DNA Methylation Signature of Post-injury Neointimal Cells During Vascular Remodeling in the Rat Balloon Injury Model. Mol Biol 5: 163. doi:10.4172/2168-9547.1000163

Page 2 of 8

mounted onto glass slides and PET FrameSlide (Cat. No. 11505151, Leica Microsystems). The sections were briefly stained by hematoxylin/ eosin and air-dried at room temperature.

\section{Isolation of neointimal cells by laser microdissection}

A Leica LMD 7000 Laser Microdissection System (Leica Microsystems) was used to isolate cells from the tissue sections. The tissues were visualized under a microscope, target cells were selected and encircled on the computer monitor using a mouse, and then the computer program guided a UV laser (337-nm wavelength) to cut the slide foil with the target cells (Figure 1). The analysis of matched tissue from the same rat avoids confounding effects of the genetic background, previously described as methylation quantitative trait loci [31]. The targeted cells fell into the $0.5-\mathrm{ml}$ thin-wall DNase-free PCR tube caps (BIO plastics) filled with $12 \mu \mathrm{l}$ DNase/RNase free water (Invitrogen, 75-0024) located beneath the visualized tissue section. The dissection conditions were optimized to obtain a clean, narrow excision of the selected cells: $40-\mathrm{XT}$ objective at power 35 to 45 and speed 3 to 4. Collected samples were centrifuged at full speed $(>10,000 \times g)$ for 5 minutes, and stored at $-20^{\circ} \mathrm{C}$. In the injured arteries, we used the laser to specifically isolate VSMC-like layer in the neointima leaving the endothelial layer intact; we also isolated the resident VSMC population in the medial layer as the control.

\section{Isolation of BMMC and PBMC}

The bone marrow mononuclear cells (BMMCs) and peripheral blood mononuclear cells (PBMCs) were isolated from whole blood and bone marrow aspirate by centrifugation through density gradient centrifugation using Histopaque 1083 (Sigma-Aldrich) as described previously [32]. To isolate PBMCs, about $0.4 \mathrm{ml}$ of blood was collected from each rat using a syringe via aortic puncture. A 21-gauge needle was directed into the thoracic cavity under the xiphoid cartilage while raising the cartilage slightly with the index finger, at approximately a $30-35^{\circ}$ angle slightly to the left of the midline. The plunger pulled back on the syringe to create a vacuum. Blood was collected into 8-ml tubes (Becton Dickinson Vacutainer Systems) containing EDTA. Whole blood was centrifuged at 3,500 rpm for $5 \mathrm{~min}$. The entire plasma layer except $5 \mathrm{~mm}(0.5 \mathrm{~mL})$ was removed. The collection tube was then tilted to a $45^{\circ}$ angle and PBMCs was removed from $2 \mathrm{~mm}$ above the interface between buffy coat layer and RBC layer. It was then added to $3.5 \mathrm{~mL}$ of PBS in $15 \mathrm{ml}$ centrifuge tube and mixed gently. About $5 \mathrm{~mL}$ of dilute buffy coat was then layered on top of $5 \mathrm{~mL}$ of HISTOPAQUE 1083 surface in a separate $15 \mathrm{~mL}$ centrifuge tube.

To isolate BMMCs, the tibia and femur bones were exposed and cut at the articulatio coxae and talotarsalis, leaving both condyli intact, and keeping the femur and tibia connected. The isolated bone was transferred into a sterile 6 -cm Petri dish, filled with $6 \mathrm{~mL}$ of cold PBS. The femurs and tibias were then separated by cutting the bone at the articulatio genus, leaving the condyli of both the tibia and femur intact. The ends of the tibia and femur were then cut away. Bone marrow cells were harvested from the male inbred SD rats by flushing tibias and femurs with cold PBS using a sterile 21-gage needle, the samples were passed through a 20.5-gage needle to disperse clumps of cells, and then through a $70-\mu \mathrm{m}$ filter to further remove bone fragments and cell clumps. Bone marrow cell suspension was transferred to a $15-\mathrm{mL}$ conical tube in cold MACS buffer and spun for $5 \mathrm{~min}$ at $675 \mathrm{~g}$ and $4^{\circ} \mathrm{C}$. The supernatant was carefully discarded and the pellet resuspended in $6 \mathrm{~mL}$ of MACS buffer at room temperature. About $6 \mathrm{ml}$ of the homogenized bone marrow cell suspension was then layered onto the HISTOPAQUE 1083 surface in a separate $15 \mathrm{ml}$ conical tube.

Histopaque layered buffy coat suspension and bone marrow aspirate suspensions were centrifuged at $400 \mathrm{~g}$ for $30 \mathrm{~min}$. Then the upper layer was aspirated, then the tube was tilted at $45^{\circ}$ and the mononuclear cell layer was taken and then added to $10 \mathrm{~mL}$ of MACS buffer $+2 \%$ FBS in
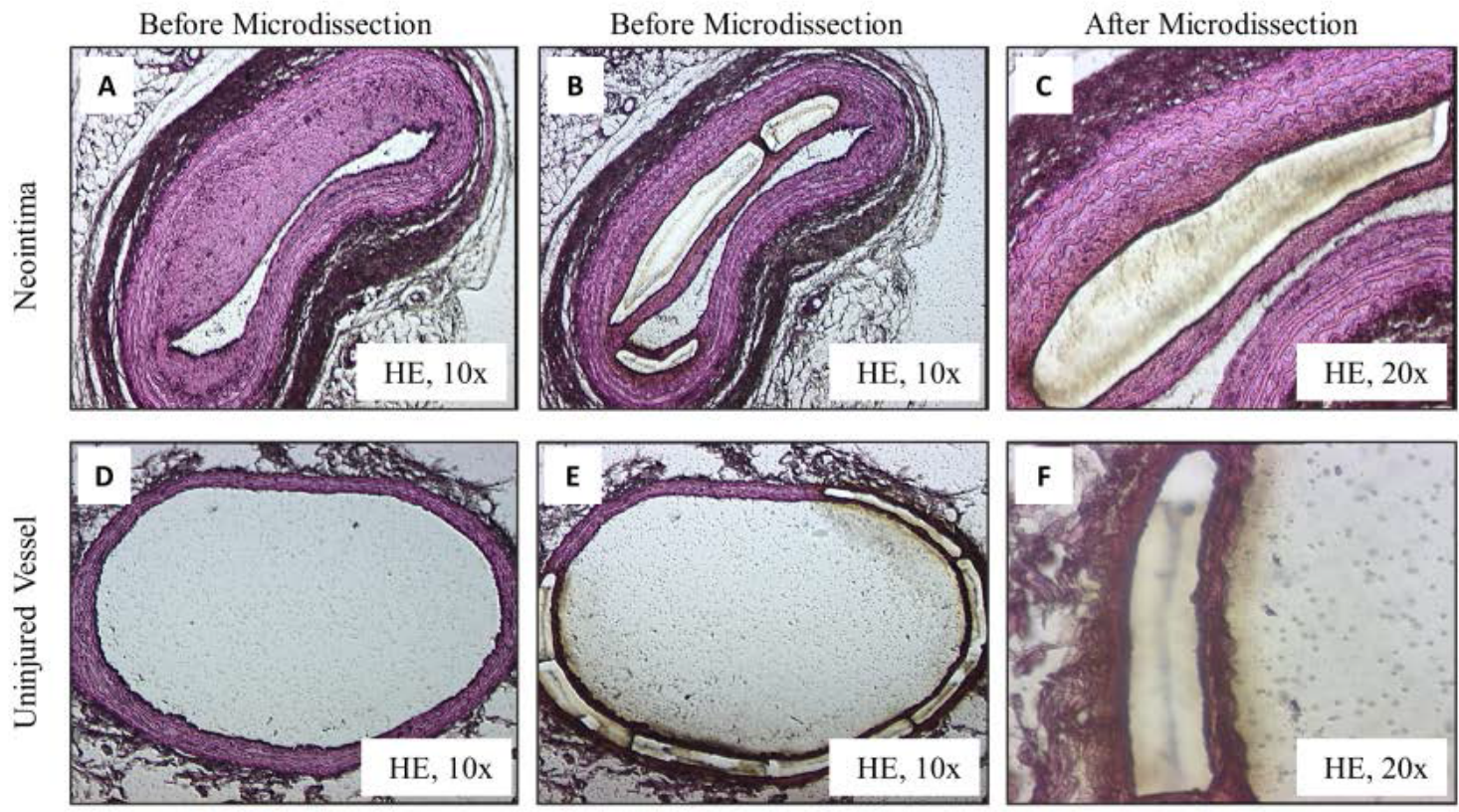

Figure 1. Laser microdissection of normal uninjured arteries and balloon injured arteries. A. The balloon injured left carotid artery before laser microdissection (10x). There is an intimal thickening and clear border between the normal intimal layer and the newly formed neointima. B-C. The balloon injured left carotid artery after microdissection of neointimal cells (10x and 20x)

D. The normal uninjured right carotid artery before microdissection (10x).

E-F. The normal uninjured right carotid artery after microdissection (10x and 20x). 
$15 \mathrm{~mL}$ centrifuge tube and gently mixed. Tubes were spun at $300 \mathrm{~g}$ for $7 \mathrm{~min}$, and the cell pellet was resuspended in $10 \mathrm{ml}$ of MACS buffer .

\section{Isolation of CD14+ BMMC using MACS}

BMMCs were further isolated by CD14 positive using Magnetic Affinity Cell Sorting (MACS). All reagents and supplies for MACS separation were purchased from Miltenyi Biotec, Bergisch-Gladbach, Germany. MACS buffer and instruments were pre-cooled to $4^{\circ} \mathrm{C}$ prior to use. About $1 \times 10^{7}$ BMMCs were centrifuged at $300 \mathrm{~g}$ for $10 \mathrm{~min}$ at $4{ }^{\circ} \mathrm{C}$. The supernatant was aspirated and cell pellet was resuspended into $100 \mu \mathrm{l}$ of cold MACS buffer. Then $2 \mu \mathrm{l}$ of biotin conjugated rabbit anti-CD14 antibody (Bioss, bs-1192R-Biotin) was added, and incubated at $4^{\circ} \mathrm{C}$ for $10 \mathrm{~min}$. Cells were washed with $2 \mathrm{ml}$ of cold MACS buffer and centrifuged. The supernatant was aspirated completely and the cell pellet was resuspended in $80 \mu \mathrm{l}$ of cold MACS buffer. Magnetic labeling of CD14 labeled BMMCs with Anti-Biotin MicroBeads (Miltenyi Biotec, 130-090-485) and positive selection of CD14+ cells was performed following the manufacturer's instructions. The isolated cell fraction was passed over a new, freshly prepared column according to Miltenyi protocol to increase the purity.

\section{ViaCount by Guava}

The Guava $^{\oplus}$ ViaCount $^{\oplus}$ assay was used to measure the number of BMMCs and PBMCs. During density gradient centrifugation, BMMCs were re-suspended in $10 \mathrm{~mL}$ of MACS buffer, and $20 \mu \mathrm{l}$ of cell suspension was added to $180 \mu \mathrm{L}$ of ViaCount solution (Guava Technologies 4000-0040) in a 96-well tray removed for ViaCount. The samples were vortexed and incubated, shielded from light, for 8-10 minutes at room temperature.

\section{Whole genome amplification, library preparation and bisulfite sequencing}

Genomic DNA was extracted from PBMCs and BMMCs using the Qiagen DNeasy Blood and Tissue Kit (Qiagen, 69506) according to the manufacturer's instructions. DNA concentration was measured by the Nanodrop spectrophotometer (Thermo Scientific). The collected neointimal cells and residential VSMCs were directly processed to bisulfite conversion without DNA extraction. The EZ DNA MethylationDirect ${ }^{\text {tix }}$ Kit (ZYMO RESEARCH, D5021) was used to perform bisulfite conversion. For the microdissected samples, $13 \mu \mathrm{l}$ M-Digestion Buffer and $1 \mu \mathrm{l}$ Proteinase $\mathrm{K}$ were added to $12 \mu \mathrm{l}$ of sample for digestion. The sample was incubated for $4 \mathrm{~h}$ at $50^{\circ} \mathrm{C}$. For the BMMCs, we employed $350 \mathrm{ng}$ of genomic DNA for optimized bisulfite conversion. The manufacturer's protocol for bisulfite conversion was then followed for all samples. After bisulfite conversion, the DNA was bound to a Zymo spin column and desulfonated on the column using M-desulfonation reagent per manufacturer's instructions. The bisulfite-converted DNA was eluted from the column in $10 \mu \mathrm{l}$ of water. Bisulfite converted DNA was amplified with The Single Cell Bisulfite Whole Genome Amplification \& Library Preparation Kit (Omigenomics) following the manufacturer's procedure. The products are sequencing-ready library for Illumina sequencer GAII and HiSeq2000. Quantitative PCR was used to measure the concentration of viable sequencing template molecules in the library prior to sequencing. Libraries were quantitated and spiked in $40 \%$ Phix prior to loading on an Illumina paired end flow cell (v4), and sequenced on the Illumina Genome Analyzer IIx (Illumina) at a concentration of $11 \mathrm{pM}$ with $101 \mathrm{bp}$ paired read length (paired-end sequencing PE100) as per manufacturer's instructions. Image analysis and base calling was performed by Illumina Real Time Analysis (RTA) v1.9.35 and output of RTA was demultiplexed and converted to Fastq format with Illumina CASAVA 1.8.2.

\section{Bioinformatic analysis}

We downloaded the rat genome sequence (RGSC 5.0/rn5 assembly) from the University of California Santa Cruz Genome Bioinformatics Site (http://genome.ucsc.edu). Sequence reads were mapped to the rat converted reference genome using the software package BSMAP [33] Fisher's exact test was used to evaluate the significance of differential methylation at a single base level. A base was deemed to be differentially methylated if the difference between the mean $\beta$ value of two sample groups (i.e., case vs control) was $>=0.5$ and the adjusted $p$-value from Fisher's exact test (false discovery rate correction) was 0.01 . The sliding linear model (SLIM) method was used to adjust the observed p-values to q-values [34]. To associate the DMS to a gene region, we computed the distance to the nearest transcription start site (TSS). We did not use cut off (say, TSS distance $=\mu$ ) to exclude putative genes for downstream analysis such as enrichment analysis with IPA (Ingenuity Pathway Analysis). DMR analysis was done at single base level. IPA was used for canonical pathway analyses of those validated, differential genes. This bioinformatics tool was used to provide insights into the most involved biological pathways in arterial remodeling based on DNA methylation alterations. Three publicly available rat whole-genome bisulfite sequencing datasets were downloaded from NCBI's Gene Expression Omnibus (GSE19830). These datasets included liver [35], brain [36], and mammary tissue [37] (GSE50077), mammary gland (GSE40251) and the liver (GSE31571). All SRA files were converted to FASTQ format using the 'fastq-dump utility in the SRA toolkit. They were analyzed using the same pipeline as described above.

\section{Results}

\section{Global DNA methylation patterns}

We obtained whole-genome bisulfite sequencing data from neointimal cells, residential vascular smooth muscle cells (VSMC), peripheral blood mononuclear cells (PBMCs) and bone marrow mononuclear cells (BMMCs). We generated totally 13,968,170 reads, $13,197,332$ reads, 9,067,668 reads, and 11,116,242 reads for BMMCs and PBMCs, neointimal cells, and VSMCs, respectively. We counted the conversion rates at non- $\mathrm{CpG}$ cytosines of those uniquely mapped reads. The results are $98.6 \%$ (neointimal), 98.4\% (VSMCs), 99.3\% (BMMCs), and $99.2 \%$ (PBMCs). Whole genome methylation sequencing was complemented by publicly available whole-genome bisulfite sequencing data, including mammary tissue, brain and liver.

In general, the neointimal cells showed an overall global methylation percentage similar to the BMMC/PBMC samples (Figure 2). Although the resident VSMCs showed a lower overall $\mathrm{CpG}$ methylation rate, they contained more highly ( $>90 \%)$ methylated CpGs $(65.3 \%)$ than the neointimal cells (59.5\%), PBMCs (57.5\%) and BMMCs (55.4\%). Quantification of average methylation for individual CpGs confirms the normal bimodal distribution of methylation rate in normal cells denoting that the majority of bases are either 'largely unmethylated' ( $<20 \%$ of reads showing methylation) or 'largely methylated' $(>80 \%$ of reads) (Figure 3).

We determined which cell types resembles the neointimal cells the most on the methylation profile. We calculated pairwise Pearson correlation coefficients between the neointimal cells and residential VSMC, BMMC and PBMC. The Pearson Correlation coefficients were then used to perform hierarchical clustering of samples based on their molecular signatures. Samples were clustered hierarchically using "correlation" as the distance metric and "Ward's method" as the agglomeration method in the clustering algorithm. The results showed that the epigenetic signature of neointimal cells were more similar to the 
Citation: Richards J, Ogoe HA, Li W, Babayewa O, Xu W, et al. (2016) DNA Methylation Signature of Post-injury Neointimal Cells During Vascular Remodeling in the Rat Balloon Injury Model. Mol Biol 5: 163. doi:10.4172/2168-9547.1000163

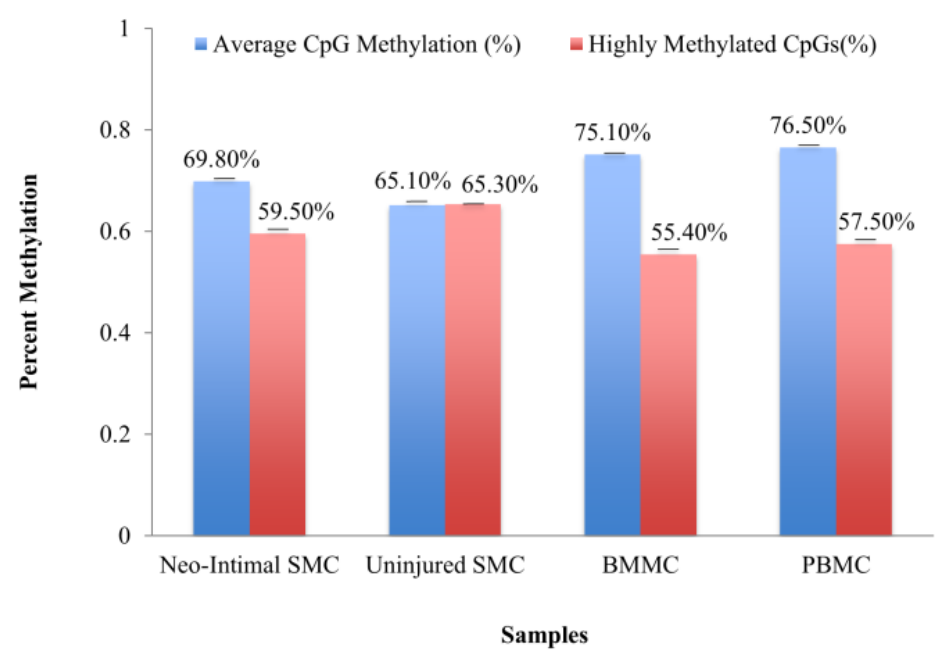

Figure 2. Global CpG methylation analysis. Averaged CpG methylation rate and the percentage of highly methylated CpGs are shown for neointimal cells, resident VSMCs, PBMCs and BMMCs. The average methylation shows the ratio of the CpG methylated dinucleotides in each sample to the total number of matched reads. The highly methylated percentage shows the percentage of methylated $\mathrm{CpGs}$ with b-values $>90 \%$.

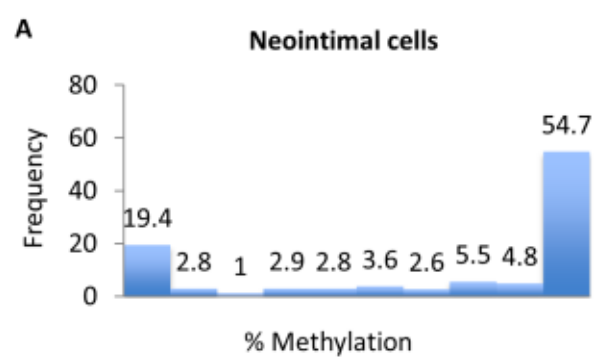

C

PBMCs

B

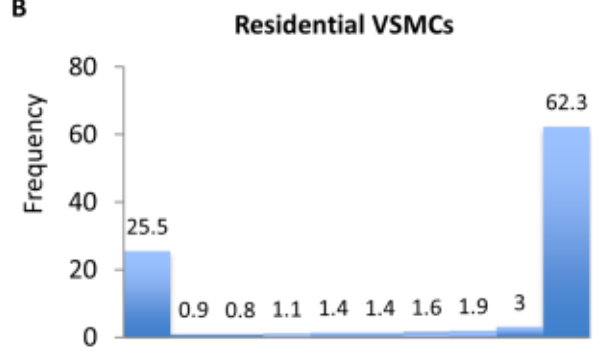

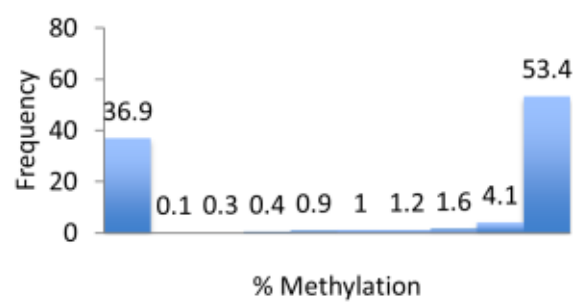

D

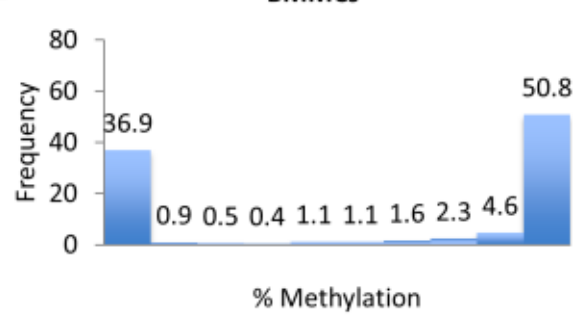

Figure 3. Histograms of methylation percentage per cytosine. The distribution of methylation levels (\%) across all CpGs is shown for neointimal cells (A), residential VSMCs (B), PBMCs (C) and BMMCs (D). Methylation levels are bimodal denoting that the majority of bases have either high or low methylation.

BMMC and BMC than to residential VSMC (Figure 4). It is noteworthy that PBMCs and BMMCs were very close even they were obtained from different donors.

\section{Differential methylation sites}

We searched for differential methylation sites (DMS) by comparing the $\beta$-values (methylation values ranging from 0.0 to 1.0 ) in the neointimal cells with residential cells, $\mathrm{PBMC}$ and $\mathrm{BMMC}$ samples. For calling a CpG site as differentially methylated (single methylation polymorphisms, SMPs), we required a minimum absolute $\Delta \beta$-value of 0.5 and a false discovery rate (FDR)-adjusted Wilcoxon rank-sum $\mathrm{P}$-value of $<0.01$ for the difference. The relatively specific SMPs for different cell types are shown in (Supplementary Table 1). In total, 2,360 of the DMS annotated to 1,127 gene regions, among which $52.8 \%$ of DMS were hypermethylated in the neointimal samples compared to the resident VSMCs.

\section{Ingenuity pathway analysis}

To explore putative functional roles for the DMS, we performed gene set enrichment analysis using Ingenuity Pathway Analysis tool. Among those genes containing DMS between the neointimal cells and residential VSMCs, 61 DMS were found to be associated with 38 genes, mainly in functional classes, such as cardiovascular system development, cell growth and proliferation, cell death and survival, embryonic development, cellular movement, and skeletal and muscular system development (Supplementary Table 2). The neointimal cells shared 72 DMS on 40 genes with BMMC and PBMC, which are mainly in the systems of cell-mediated immune response, hematological system development and function, embryonic development, inflammatory response.

\section{Differentially methylated regions (DMRs)}

Because the PBMCs and BMMCs had very similar DNA 
methylation profiles, they were combined and analyzed as a single set. We interrogated the non-repetitive part of the genome for regions containing at least five consecutive and consistently differentially methylated CpGs and in which DNA methylation levels of the flanking $\mathrm{CpG}$ sites differed significantly between the two samples (Fisher's exact test, $\mathrm{q}<0.05$ and percent methylation difference larger than 20\%). Using these criteria, we identified 662 DMRs between neointimal cells and residential VSMCs, 69 DMRs between neointimal cells and BMMC/ PBMC (Supplementary Table 3). DMRs were mostly hypermethylated in the neointimal cells compared with residential VSMCs (Supplementary

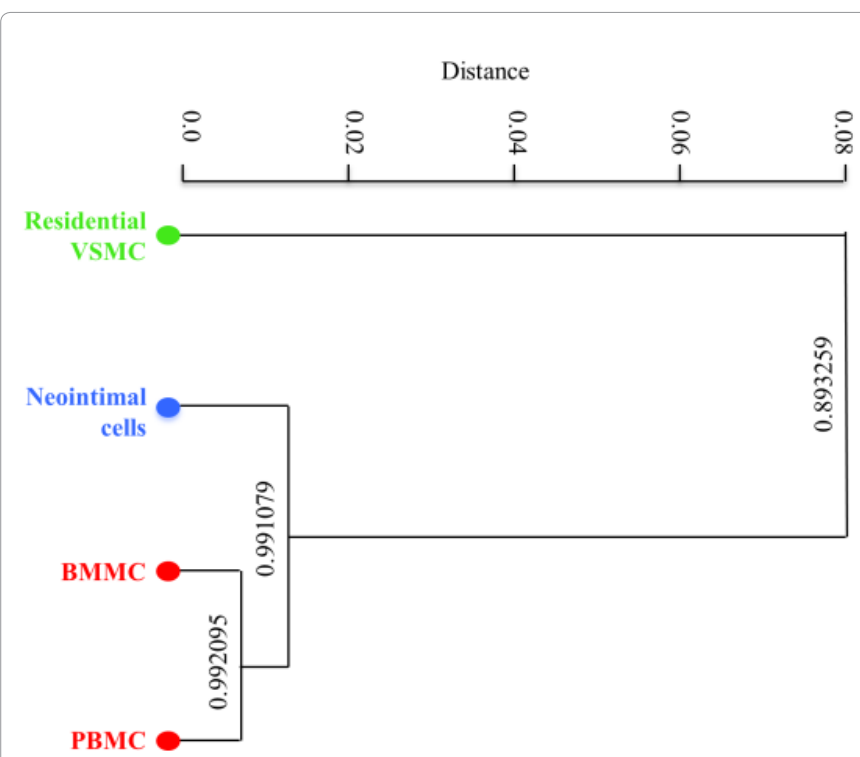

Figure 4. Hierarchical clustering of the neointimal cells, residential VSMCs, PBMCs and BMMCs using Pearson's correlation distance and "Wards method". Pair-wise Pearson's correlation scores are shown in the chart.
Table 4). The majority of DMRs were located in intergenic regions, outside those CpG islands and island shores (Figure 5). Interestingly, exons have less DMRs than promotors and introns, and $\mathrm{CpG}$ islands contain more DMRs than islands shores (Figure 5).

\section{Biological relevance of differentially methylated regions}

We used 662 genes corresponding to the DMRs between the neointimal cells vs. residential VSMCs to perform gene set enrichment analysis using Ingenuity Pathway Analysis. We excluded all regions that were also found to be differentially methylated in other pairwise comparisons (Figure 6). Totally, 423 genes were found in the DMRs unique to the comparison between neointimal cells and residential VSMCs (Supplementary Table 5). In this analysis, DMRs that appear in the pairwise comparisons between other cell types in this study were excluded. Furthermore, gene set enrichment analysis showed that the DMRs between neointimal cells and residential VSMCs mainly corresponded to genes in the stem cell pluripotency, cell differentiation, proliferation, and self-renewal categories according to the ranking of statistical significance among various functional categories based on $-\log (P$ value) (Supplementary Table 6$)$. Other high scoring gene networks include hematologic system development and function, and cardiovascular system development and function. The genes in the DMRs that relate to the regulation of smooth muscle differentiation, VSMC development, and stem cell maintenance and differentiation are provided in (Supplementary Table 7).

\section{Discussion}

In this study, we compared the DNA methylation profiles between neointimal cells, residential VSMCs and bone marrow derived circulating progenitor cells. The results showed a clear clustering of the neointimal cells with the PBMCs/BMMCs.

It is known that adult vascular smooth muscle cells (VSMCs) proliferate at a very low rate, exhibit very low synthetic activity, and express a unique set of markers [38]. These cells retain remarkable

C Neointimal cells vs. Residential VSMC

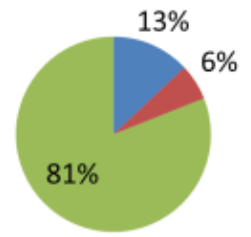

D Neointimal cells vs. BMMC/PBMC

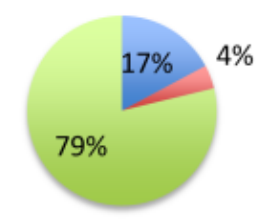

CpG islands

- Shores $\quad$ Other

Figure 5. Annotations of differentially methylated CpG sites. (a-b) Percentages of differentially methylated CpG sites on promoters, exons, introns and intergenic regions. (c-d) Percentages of differentially methylated $\mathrm{CpG}$ sites on $\mathrm{CpG}$ islands, CpG island shores (defined as $2 \mathrm{~kb}$ flanks of $\mathrm{CpG}$ islands) and all other regions outside of shores and $\mathrm{CpG}$ islands. 


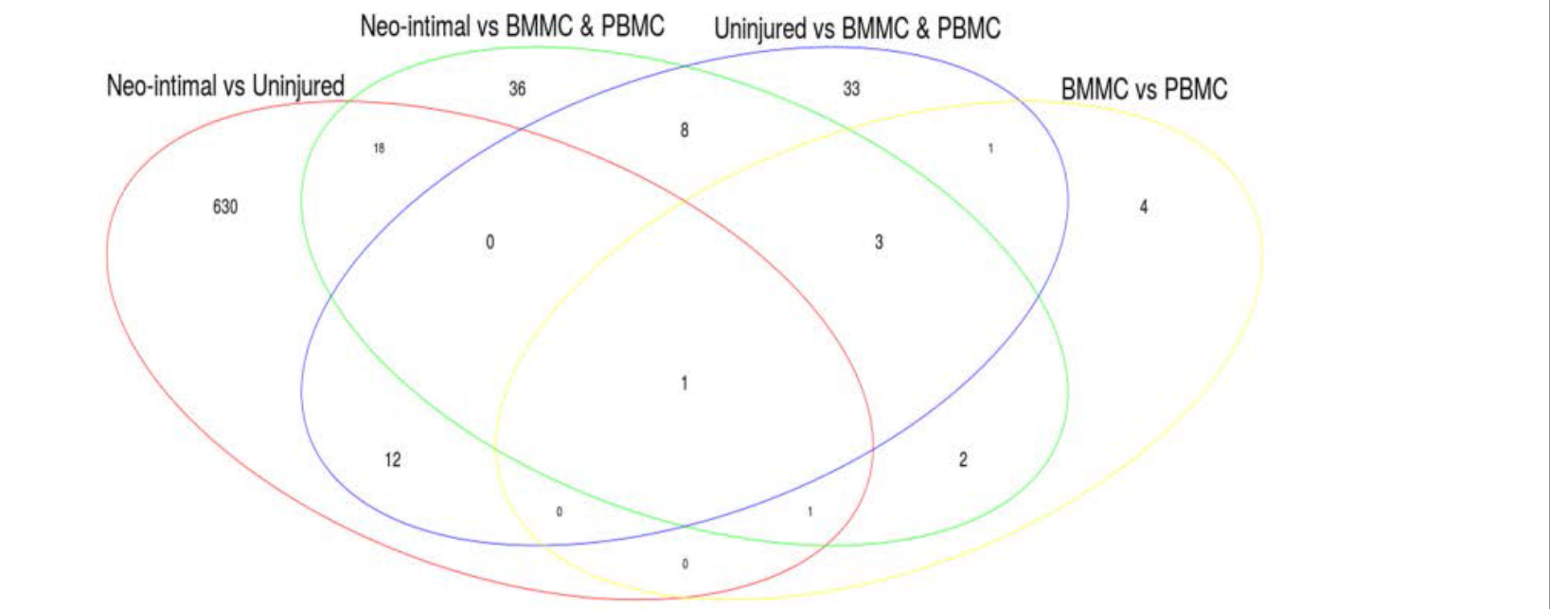

Figure 6: Venn diagram showing differentially methylated regions common and unique to comparisons between different cell lineages.

plasticity and can undergo profound phenotypic transition in response to local environmental changes ${ }^{17}$. When blood vessel is injured, VSMCs dramatically increase their proliferation, migration, and synthetic capacity; however, the high degree of plasticity can also lead to an adverse phenotypic switch and vascular diseases, such as atherosclerosis, restenosis, cancer, and hypertension [38,39]. VSMC phenotypic modulation has been a research focus in recent years. It has been discovered that Klf4 plays a critical role in the phenotypic transitions of VSMCs that have favorable effects in inhibiting plaque pathogenesis [16]; and a microRNA gene (miR-133) also appears to be a key regulator of VSMCs phenotypic switch in vitro and in vivo [40]. There are some evidences suggesting that neointimal cells mostly derive from vascular cells; although the BM-derived monocytes/ macrophages were abundant in the neointima at 7 days after balloon injury, they almost disappeared at 30 days post-surgery [41-46]. Furthermore, a recent study that used Myh11-CreER ${ }^{\mathrm{T} 2}$ ROSA floxed STOP eYFP Apoe-/- mice for SMC lineage tracing showed that $>80 \%$ of SMCs within advanced atherosclerotic lesions are phenotypically modulated and that traditional methods for detecting SMCs based on immunostaining for SMC markers fail to detect $>80 \%$ of SMCderived cells within advanced atherosclerotic lesions, indicating that the contribution of SMCs to atherosclerotic plaques has been greatly underestimated by conventional techniques [16].

On the other hand, several studies have suggested that circulating $\mathrm{BM}$ derived cells contribute to neointima formation [13,47,48]. In chimeric mice, high-resolution confocal microscopy showed that some BM-derived cells expressed SMMHC, a specific SMC marker protein [49]; two-photon microscopy techniques observed a transdifferentiation of BM-derived cells into VSMCs [50]. In humans, a combined immunohistochemical and FISH analysis showed a substantial fraction of VSMCs throughout the atherosclerotic vessel wall that were originated from donor bone marrow in the patients receiving sex-mismatched bone marrow transplantation [51]. Recently, it has been reported that blood vessel wall contains a type of stem cells (multipotent vascular stem cells, MVSCs), which can differentiate into SMCs [52]. These studies support that SMCs in the neointima are derived from progenitors.

Traditional VSMC markers (such as a-SMA, SM-MHC or calponin) lack lineage-specificity to trace the embryonic origin of cells [38,53-57]. In addition, these markers can change during differentiation and are not suitable for tracing cell origins during trans-differentiations, dedifferentiation and differentiations. As reviewed by Owens et al, because a key feature of vascular remodeling is the loss of expression of SMCselective gene products, such as SM MHC and SM $\alpha$-actin (SMaA), there are still major ambiguities regarding the definitive identification of altered SMC phenotypes during this process [17]. Green-fluorescent protein [13,58,59] and Y chromosome [45] have been used as markers for tracking the cell origin by distinguishing donor cells from recipient cells in transplantation animal models [57,60], which may be confounded by the immune rejections of transplant models. In the stem cell differentiation and reverse differentiation studies in recent years, it has been suggested that DNA methylation patterns may provide a tool to recognize the cells because they are signatures that contain the memory of re-programmed cells even after the cells switch to another cell type [25-29]. We believe that the comparison of DNA methylation signatures can provide additional insight about the origins of neointimal cells. Our results showed a clustering of the neointimal cells with the PBMCs/ BMMCs on DNA methylation patterns. In fact, it has been reported that those unidentified SMC-derived cells also exhibit phenotypes of other cell lineages during the phenotypical SMCs transition within lesions, including macrophages and mesenchymal stem cells (MSCs) but are distinctly different from classical monocytes, macrophages, and dendritic cells $[16,61]$. Indeed, our study has some caveats. For example, we studied the cells based on their physical positions so we did not further purify the cells based on some cell markers and we did not examine purity. Thus, the collected cells may contain multiple cell types and/or transition states, so our data cannot answer the question where the neointimal cells come from, they may either be differentiated from progenitor cells (circulating or residential) or transitioned from VSMCs. However, our results can answer the question that at a late stage (such as 30th days after injury) the neointimal cells have some signatures of circulating progenitor cells. Another caveat is that our data cannot answer the question if the observation will be similar or different during the remodeling process (earlier than $30^{\text {th }}$ day). The third caveat is that the small sample size, although we isolated 50-100 cells, but they were from one rat, so we cannot eliminate the personal bias from rat to rat. Next caveat is the low coverage, which is similar to an array study, only a small number of CpG sites were studied instead of all CpG sites in the whole-genome, it may cause biases because some CpGs may be more important than others regarding the differentiation or transition during vascular remodeling. 
Citation: Richards J, Ogoe HA, Li W, Babayewa O, Xu W, et al. (2016) DNA Methylation Signature of Post-injury Neointimal Cells During Vascular Remodeling in the Rat Balloon Injury Model. Mol Biol 5: 163. doi:10.4172/2168-9547.1000163

In conclusion, we have specifically isolated neointimal cells and compared their methylation signature with the other two hypothesized cell origins; the residential VSMCs and the circulating progenitor cells. To our knowledge this is the first time that laser microdissection and methylation signatures have been used to address this issue. Although the question about the origination of neointimal cells can only be completely addressed after migration and trans-differentiation are directly observed, our study showed that neointimal cells have a more similar methylation profile to the circulating bone marrow derived cells than the residential VSMCs at the $30^{\text {th }}$ day after vascular injury.

\section{Acknowledgements}

This work was supported by National Institutes of Health (R21HG006173, R43HG007621, RC4MD005964, HL117929, U54RR003034, U54MD07588, T32HL103104, T32HL067702); and the American Heart Association grant (09GRNT2300003).

\section{Author Contributions}

J.R. and L.M. performed the microdissection and WGA experiments H.A.O. carried out the computational analysis. W.L. carried out high-throughput sequencing and read mapping. O.B. W.X. T.B. M.G.B. and Y.X. performed animal and histological experiments. J.R., M.G.B. and Q.S. wrote the manuscript. L.M. and Q.S. designed and supervised the study.

\section{References}

1. Buck RC (1961) Intimal thickening after ligature of arteries an electronmicroscopic study. Virc Res 9: 418.

2. Ross R (1999) Atherosclerosis--an inflammatory disease. N Engl J Med 340: $115-126$

3. Clowes AW, Reidy MA, Clowes MM (1983) Kinetics of cellular proliferation after arterial injury. I. Smooth muscle growth in the absence of endothelium. Lab Invest 49: 327-333.

4. Clowes AW, Schwartz SM (1985) Significance of quiescent smooth muscle migration in the injured rat carotid artery. Circ Res 56: 139-145.

5. Velican D, Velican C (1981) Comparative study on age-related changes and atherosclerotic involvement of the coronary arteries of male and female subjects up to 40 years of age. Atherosclerosis 38: 39-50.

6. Stemerman MB, Weinstein R, Rowe JW, Maciag T, Fuhro R et al. (1982) Vascular smooth muscle cell growth kinetics in vivo in aged rats. Proc Nat Acad Sci USA 79: 3863-3866

7. Soyombo AA, Angelini GD, Bryan A J, Jasani B, Newby AC (1990) Intima proliferation in an organ culture of human saphenous vein. Am J Pathol 137 1401-1410.

8. Jackman RW, Anderson SK, Sheridan JD (1988) The aortic intima in organ culture. Response to culture conditions and partial endothelial denudation. Am J Pathol 133: 241-251.

9. Jackman RW (1982) Persistence of axial orientation cues in regenerating intima of cultured aortic explants. Nature 296: 80-83.

10. Owens GK (1995) Regulation of differentiation of vascular smooth muscle cells. Physiol Rev 75: 487-517.

11. Gabbiani G, Rungger-Brändle E, de Chastonay C, Franke WW (1982) Vimentincontaining smooth muscle cells in aortic intimal thickening after endothelia injury. Lab Invest 47: 265-269.

12. Barja F, Coughlin C, Belin D, Gabbiani G (1986) Actin isoform synthesis and mRNA levels in quiescent and proliferating rat aortic smooth muscle cells in vivo and in vitro. Lab Invest 55: 226-233.

13. Sata M, Saiura A, Kunisato, Tojo A, Okada S, Tokuhisa T et al. (2002) Hematopoietic stem cells differentiate into vascular cells that participate in the pathogenesis of atherosclerosis. Nat Med 8: 403-409.

14. Sata M, Fukuda D, Tanaka K, Kaneda Y, Yashiro H, Shirakawa I (2005) The role of circulating precursors in vascular repair and lesion formation. $\mathrm{J}$ Cell Mol Med 9: 557-568.

15. Hillebrands JL, Klatter FA, Rozing J (2003) Origin of vascular smooth muscle cells and the role of circulating stem cells in transplant arteriosclerosis. Arterioscler Thromb Vasc Biol 23: 380-387.
6. Shankman LS, Gomez D, Cherepanova OA, Salmon M, Alencar GF, Haskins RM et al. (2015) KLF4-dependent phenotypic modulation of smooth muscle cells has a key role in atherosclerotic plaque pathogenesis. Nat Med 21: 628637.

17. Nguyen AT, Gomez D, Bell RD, Campbell JH, Clowes AW, Gabbiani G et al. (2013) Smooth muscle cell plasticity: fact or fiction? Circ Res 112: 17-22.

18. Gomez D \& Owens GK (2012) Smooth muscle cell phenotypic switching in atherosclerosis. Cardiovasc Res 95: 156-164.

19. Yang P, Hong MS, Fu C, Schmit BM, Su Y, Berceli SA et al. (2016) Preexisting smooth muscle cells contribute to neointimal cell repopulation at an incidence varying widely among individual lesions. Surgery 159: 602-612.

20. Herring BP, Hoggatt AM, Burlak C \& Offermanns S (2014) Previously differentiated medial vascular smooth muscle cells contribute to neointima formation following vascular injury. Vascular cell 6: 21.

21. Trowbridge JJ, Snow JW, Kim J, Orkin SH (2009) DNA methyltransferase 1 is essential for and uniquely regulates hematopoietic stem and progenitor cells. Cell Stem Cell 5: 442-449.

22. Broske AM, Vockentanz L, Kharazi S, Huska MR, Mancini E, Scheller M et al. (2009) DNA methylation protects hematopoietic stem cell multipotency from myeloerythroid restriction. Nat Genet 41: 1207-1215.

23. Bird A (2002) DNA methylation patterns and epigenetic memory. Genes Dev 16: 6-21.

24. Bergman $\mathrm{Y}$, Cedar $\mathrm{H}$ (2010) Epigenetic control of recombination in the immune system. Semin Immunol 22: 323-329.

25. Zaidi SK, Young DW, Montecino M, van Wijnen AJ, Stein JL, Lian JB et al (2011) Bookmarking the genome: maintenance of epigenetic information. J Bio Chem 286: 18355-18361.

26. Polo JM, Liu S, Figueroa ME, Kulalert W, Eminli S, Tan KY et al. (2010) Cell type of origin influences the molecular and functional properties of mouse induced pluripotent stem cells. Nat Biotechnol 28: 848-855

27. Kim K, Doi A, Wen B, Ng K, Zhao R, Cahan P et al. (2010) Epigenetic memory in induced pluripotent stem cells. Nature 467: $285-290$

28. Bar-Nur O, Russ HA, Efrat S, Benvenisty N (2011) Epigenetic memory and preferential lineage-specific differentiation in induced pluripotent stem cells derived from human pancreatic islet beta cells. Cell Stem Cell 9: 17-23.

29. Lister R, Pelizzola M, Kida YS, Hawkins RD, Nery JR, et al. (2011) Hotspots of aberrant epigenomic reprogramming in human induced pluripotent stem cells Nature 471: 68-73.

30. Gabeler EE, van Hillegersberg R, Statius van Eps RG, Sluiter W, et al. (2002 A comparison of balloon injury models of endovascular lesions in rat arteries. BMC Cardiovasc Disord 2: 16.

31. Barrachina M, Ferrer I (2009) DNA methylation of Alzheimer disease and tauopathy-related genes in postmortem brain. J Neuropathol Exp Neurol 68: 880-891.

32. Asahara T, Murohara T, Sullivan A, Silver M, van der Zee R, et al. (1997) Isolation of putative progenitor endothelial cells for angiogenesis. Science 275 964-967.

33. Xi Y, Li W (2009) BSMAP: whole genome bisulfite sequence MAPping program. BMC Bioinformatics 10: 232

34. Wang HQ, Tuominen LK, Tsai CJ (2011) SLIM: a sliding linear model for estimating the proportion of true null hypotheses in datasets with dependence structures. Bioinformatics 27: 225-231.

35. Sati S, Tanwar VS, Kumar KA, Patowary A, Jain V, Ghosh S et al (2012) High resolution methylome map of rat indicates role of intragenic DNA methylation in identification of coding region. PLoS One 7: e31621.

36. Kobow K, Kaspi A, Harikrishnan KN, Kiese K, Ziemann M, Khurana I et al. (2013) Deep sequencing reveals increased DNA methylation in chronic rat epilepsy. Acta Neuropathol 126: 741-756.

37. de Assis S, Warri A, Cruz MI, Laja O, Tian Y, Zhang B et al. (2012) High-fat or ethinyl-oestradiol intake during pregnancy increases mammary cancer risk in several generations of offspring. Nat Commun 3: 1053

38. Owens GK, Kumar MS, Wamhoff BR (2004) Molecular regulation of vascular smooth muscle cell differentiation in development and disease. Physiol Rev 84: 767-801. 
Citation: Richards J, Ogoe HA, Li W, Babayewa O, Xu W, et al. (2016) DNA Methylation Signature of Post-injury Neointimal Cells During Vascular Remodeling in the Rat Balloon Injury Model. Mol Biol 5: 163. doi:10.4172/2168-9547.1000163

39. Indolfi C, Mongiardo A, Curcio A, Torella D (2003) Molecular mechanisms of instent restenosis and approach to therapy with eluting stents. Trends Cardiovasc Med 13: 142-148.

40. Torella D, laconetti C, Catalucci D, Ellison GM, et al. (2011) MicroRNA-133 controls vascular smooth muscle cell phenotypic switch in vitro and vascular remodeling in vivo. Circ Res 109: 880-893.

41. Rodriguez-Menocal L, St-Pierre M, Wei Y, Khan S, Mateu D, et al. (2009) The origin of post-injury neointimal cells in the rat balloon injury model. Cardiovasc Res 81: 46-53.

42. Hu Y, Davison F, Ludewig B, Erdel M, Mayr M, Url M, et al. (2002) Smooth muscle cells in transplant atherosclerotic lesions are originated from recipients, but not bone marrow progenitor cells. Circulation 106: 1834-1839.

43. Hu Y, Mayr M, Metzler B, Erdel M, Davison F, et al. (2002) Both donor and recipient origins of smooth muscle cells in vein graft atherosclerotic lesions. Circ Res 91: e13-20.

44. Li J, Han X, Jiang J, Zhong R, Williams GM, et al. (2001) Vascular smooth muscle cells of recipient origin mediate intimal expansion after aortic allotransplantation in mice. Am J Pathol 158: 1943-1947.

45. Bentzon JF, Weile C, Sondergaard CS, Hindkjaer J, Kassem M (2006) Smooth muscle cells in atherosclerosis originate from the local vessel wall and not circulating progenitor cells in ApoE knockout mice. Arterioscler Thromb Vasc Biol 26: 2696-2702.

46. Bentzon JF, Sondergaard CS, Kassem M, Falk E (2007) Smooth muscle cells healing atherosclerotic plaque disruptions are of local, not blood, origin in apolipoprotein E knockout mice. Circulation 116: 2053-2061.

47. Saiura A, Sata M, Hirata Y, Nagai R, Makuuchi M (2001) Circulating smooth muscle progenitor cells contribute to atherosclerosis. Nat Med 7: 382-383.

48. Shimizu K, Sugiyama S, Aikawa M, Fukumoto Y, Rabkin E, et al. (2001) Host bone-marrow cells are a source of donor intimal smooth- muscle-like cells in murine aortic transplant arteriopathy. Nat Med 7: 738-741.

49. Kumar AH, Metharom P, Schmeckpeper J, Weiss S, Martin K, et al. (2010) Bone marrow-derived CX3CR1 progenitors contribute to neointimal smooth muscle cells via fractalkine CX3CR1 interaction. FASEB J 24: 81-92.

50. Subramanian P, Karshovska E, Reinhard P, Megens RT, Zhou Z, et al. (2010) Lysophosphatidic acid receptors LPA1 and LPA3 promote CXCL12-mediated smooth muscle progenitor cell recruitment in neointima formation. Circ Res 107: 96-105.

51. Caplice NM, Bunch TJ, Stalboerger PG, Wang S, Simper D, et al. (2003) Smooth muscle cells in human coronary atherosclerosis can originate from cells administered at marrow transplantation. Proc Natl Acad Sci U S A 100: 4754-4759.

52. Tang Z, Wang A, Yuan F, Yan Z, Liu B, et al. (2012) Differentiation of multipotent vascular stem cells contributes to vascular diseases. Nat Commun 3: 875

53. Hoofnagle MH, Thomas JA, Wamhoff BR, Owens GK (2006) Origin of neointimal smooth muscle: we've come full circle. Arterioscler Thromb Vasc Biol 26: 2579-2581.

54. Jabs A, Moncada GA, Nichols CE, Waller EK, Wilcox JN (2005) Periphera blood mononuclear cells acquire myofibroblast characteristics in granulation tissue. J Vasc Res 42: 174-180.

55. Sugiyama S, Kugiyama K, Nakamura S, Kataoka K, Aikawa M, et al. (2006) Characterization of smooth muscle-like cells in circulating human peripheral blood. Atherosclerosis 187: 351-362.

56. Tanaka K, Sata M (2009) Role of vascular progenitor cells in cardiovascular disease. Curr Pharm Des 15: 2760-2768.

57. Tanaka K, Sata M, Natori T, Kim-Kaneyama JR, Nose K, et al. (2008) Circulating progenitor cells contribute to neointimal formation in nonirradiated chimeric mice. FASEB J 22: 428-436.

58. Wang $\mathrm{CH}$, Anderson N, Li SH, Szmitko PE, Cherng WJ, et al. (2006) Stem cell factor deficiency is vasculoprotective: unraveling a new therapeutic potential of imatinib mesylate. Circ Res 99: 617-625.

59. Zernecke A, Schober A, Bot I, von Hundelshausen P, Liehn EA, et al. (2005) SDF-1alpha/CXCR4 axis is instrumental in neointimal hyperplasia and recruitment of smooth muscle progenitor cells. Circ Res 96: 784-791.

60. Tanaka K, Sata M, Hirata Y, Nagai R (2003) Diverse contribution of bone marrow cells to neointimal hyperplasia after mechanical vascular injuries. Circ Res 93: 783-790.

61. Vengrenyuk Y, Nishi H, Long X, Ouimet M, Savji N, et al. (2015) Cholestero loading reprograms the microRNA-143/145-myocardin axis to convert aortic smooth muscle cells to a dysfunctional macrophage-like phenotype. Arterioscler Thromb Vasc Biol 35: 535-546. 